\title{
Triblock Copolymers in a Selective Solvent. 2. Semidilute Solutions
}

\author{
Eric Raspaùd, Didier Lairez,* and Mireille Adam \\ Laboratoire Léon Brillouin, CEA-CNRS, CE-Saclay, 91191 Gif-sur-Yvette Cedex, France
}

\author{
Jean-Pierre Carton
}

Service de Physique de l'Etat Condensé, CE-Saclay, 91191 Gif-sur-J'vette Cedex, France

Received August 10, 1995; Revised Manuscript Received November 21, $1995^{\circledR}$

\begin{abstract}
Triblock copolymers polystyrene-polyisoprene-polystyrene are studied in semidilute solutions $\left(1<C / C^{*}<8\right)$, in a selective solvent, i.e., a nonsolvent for polystyrene and a good solvent for polyisoprene. The mass of the copolymer is equal to $1.6 \times 10^{5} \mathrm{~g} / \mathrm{mol}$ and it contains $70 \%$ of polyisoprene. Static light scattering, small-angle neutron scattering, viscoelastic, and quasi-elastic light scattering experiments are reported as a function of the concentration and of the temperature. Rheological properties argue in favor of a network made of end-linked copolymers, the cross-link dynamics being governed by the glass transition of the polystyrene terminal blocks. Small-angle neutron scattering experiments revesl that this network is well ordered and has a cubic structure as up to five Bragg peaks are olsserved. The relaxation function of concentration fluctuations measured by quasi-elastic light scattering is somewhat reminiscent of supercooled liquid and colloids near the glass transition. It is interpreted in terms of polystyrene node cooperative diffusion which becomes anomalous at long times (low $q$ ).
\end{abstract}

\section{Introduction}

Block copolymers are nowadays at the center of interest for many scientists, from both fundamental and applied points of view. In the bulk state ${ }^{1}$ as well as in selective solvent solutions, ${ }^{2,3}$ i.e., a good solvent for one block and a bad or nonsolvent for another block, segregation effects are responsible for a wide range of structures depending on the number of blocks, their degree of polymerization, their incompatibility, and the solvent quality. On the one hand, in the bulk the understanding of these systems and of their phase diagrams has widely progressed during the last decade. Macrolattice formation is extensively studied both theoretically and experimentally. On the other hand, in dilute solution aggregation processes are still an open question. This paper is concerned with semidilute solutions of block copolymers. This regime is interesting not only because it corresponds to the intermediate state between dilute aggregates and bulk macrolattice but also because of its original static and dynamic properties. These latter are the first and most visible aspects of these systems that attract physicists' attention and industrial applications. For example, polystyrenepolyisoprene-polystyrene (PS-PI-PS) block copolymers in semidilute solutions in tetradecane or heptane pass from a liquid to a solid state when the temperature decreases from 40 to $5{ }^{\circ} \mathrm{C}$, even though the material contains $90 \%$ solvent. Surprisingly, very few experiments are reported on such systems and among them, to our knowledge, static and dynamic properties were never confronted. In a previous paper, ${ }^{4}$ the aggregation of triblock copolymers PS-PI-PS in dilute solution in $n$-heptane, that is, a nonsolvent of the polystyrene terminal block, was studied at temperatures above 30 ${ }^{\circ} \mathrm{C}$. Results plead in favor of loose and polydisperse aggregates ("animals") rather than spherical micelles. In this paper, static light scattering, small-angle neutron, viscoelastic, and quasi-elastic light scattering results are reported as a function of the concentration and of the temperature in the semidilute regime.

${ }^{\otimes}$ Abstract published in Advance ACS Abstracts, January 15, 1996.

$0024-9297 / 96 / 2229-1269 \$ 12.00 / 0$

\section{Samples and Experimental Devices Characteristics}

The triblock copolymer polystyrene--polyisoprenepolystyrene studied in this paper was kindly provided by DEXCO and is known under the name VECTOR 4111D. Its polydispersity index was given to be less than 1.02, and the weight ratios of the blocks are 0.153 , 0.694 , and 0.153 , respectively. Some measurements have been performed on diblock copolymers polystyrenepolyisoprene provided by PSS Laboratories, which corresponds to half of triblock copolymers here studied (weight-average molecular mass $M_{\mathrm{W}}=8.0 \times 10^{4} \mathrm{~g} / \mathrm{mol}$, weight ratio of polystyrene 0.275 , polydispersity 1.03 ).

The homemade light scatteririg device uses a polarized light source $\left(\mathrm{Ar}^{+}\right.$, wavelength $\lambda_{0}=488 \mathrm{~nm}$ ) and a goniometer that allows measurements in the range of scattering angle $\theta$ from $15^{\circ}$ to $150^{\circ}$. The available transfer vector $\mathrm{q}=(4 \pi / \lambda) \sin (\theta / 2)$ range is thus comprised between $5 \times 10^{-3}$ and $3.6 \times 10^{-2} \mathrm{~nm}^{-1}$. Quasielastic light scattering experiments were performed using the self-beat technique, the time-averaged autocorrelation function of the scaltered intensity being obtained using a Malvern 7032 multicorrelator. Smallangle neutron measurements were done using the Pace spectrometer in Saclay at different temperatures and concentrations ( $\lambda=10 \AA$, and the distance between sample and detectors being equal to $3.2 \mathrm{~m}$ ). This spectrometer allows a transfer vector $q$ resolution of the order of $10 \%$. On one sample and at room temperature, the Pace spectrometer was used in a "time-of-flight" configuration, allowing the scattered intensity to be measured over two decades in transfer vector, with a better resolution $(\Delta \lambda / \lambda=1 \%)$. Viscoelastic measurements were performed using the magnetorheometer, ${ }^{5}$ which allows us to measure zer'o shear viscosity and longest relaxation time of the solutions in a hermetically closed cell avoiding solvent evaporation.

In $n$-heptane solutions, three cifferent concentration regimes are observed. In Figure 1, the measured light scattered intensity per monomer at zero angle reduced by its zero concentration limit $(I / C)_{q \rightarrow 0} /(I / C)_{q \rightarrow 0, C \rightarrow 0}$ is plotted as a function of the copolymer concentration $C$. At a concentration below the critical aggregation con- 


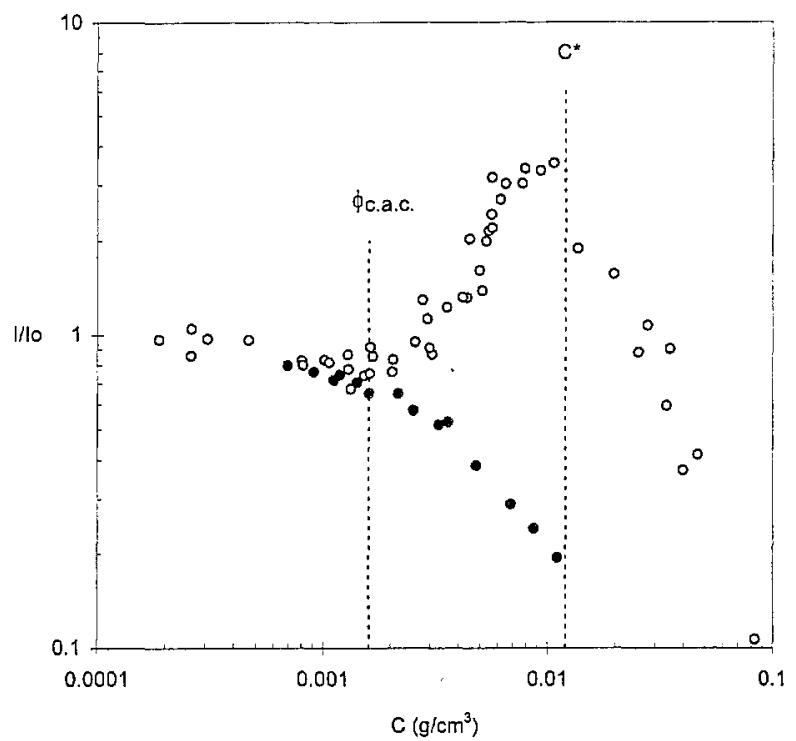

Figure 1. Light scattered intensity per monomer at zero angle reduced by its zero concentration limit $I / I_{0}=(I / C)_{q \rightarrow 0} /(I /$ $C)_{q \rightarrow, C \rightarrow 0}$ as a function of the copolymer concentration $C$. $\phi_{\mathrm{car}}$ corresponds to the concentration at which aggregates appear while $C^{*}$ is their overlap concentration. Open and filled symbols correspond to selective (heptane) and good (THF) solvents, respectively.

centration, $\phi_{\text {cac }}=1.6 \times 10^{-3} \mathrm{~g} / \mathrm{cm}^{3}$, the scattered intensity per monomer decreases slowly with the con. centration, which indicates a small positive second viria] coefficient. Extrapolation to zero concentration of light. scattering measurements in this concentration rang $\epsilon$ allows us to determine weight-average molecular weigh1. and apparent radius of gyration in good agreement witk the values determined in good solvent: $M_{\mathrm{w}}=(1.6 \pm 0.1)$ $\times 10^{5} \mathrm{~g} / \mathrm{mol}$ and $R_{\mathrm{g}}=25 \pm 2 \mathrm{~nm}$. The diffusior. coefficient determined by quasi-elastic light scattering; measurements is found to be equal to $D=(7.3 \pm 0.3) \times$ $10^{-7} \mathrm{~cm}^{2} / \mathrm{s}$ leading to an hydrodynamic radius $R_{\mathrm{h}}=10 . \varepsilon_{i}$ $\pm 0.5 \mathrm{~nm}$.

At concentration above $\phi_{\text {cac }}$, the scattered intensity per monomer $(I / C)$ increases strongly with the concen. tration and this is interpreted as being due to the growth process of triblock aggregates. Around $C=1.2 !$ $\times 10^{-2} \mathrm{~g} / \mathrm{cm}^{3}$, the scattered intensity per monomer start:; to decrease with increasing concentration due to the screening of concentration fluctuations, as expected for. semidilute solutions around $C^{*}$. This overlap concen. tration of the aggregates is comparable to the overlap) concentration of free copolymers.

In $n$-heptane at room temperature, the diblock copolymer forms spherical and starlike micelles having! an association number equal to $47 \pm 1$ and a hydrodynamic radius equal to $29 \pm 1 \mathrm{~nm}$. These results and a comparison with triblock copolymer aggregation will b: published in a forthcoming paper. ${ }^{6}$

\section{Viscoelastic Properties}

Semidilute solutions of triblock copolymer PS-PI-PS samples in $n$-heptane encounter drastic changes in. their viscoelastic properties with the temperature. Al $50^{\circ} \mathrm{C}$, these solutions present a very low viscosity thal, is about 1 order of magnitude higher than the solvent viscosity $\eta_{\mathrm{s}}$. While at $0{ }^{\circ} \mathrm{C}$, polymer solutions look liks a solid. At first sight, such a transition could bs reminiscent either of physical gelation ${ }^{7,8}$ or of glas $\$$ transition. 9 In order to quantify this aspect, measure. ments of the zero shear viscosity, $\eta$, and of the longesi, relaxation time, $\tau_{R}$, were done. Figure 2 shows the

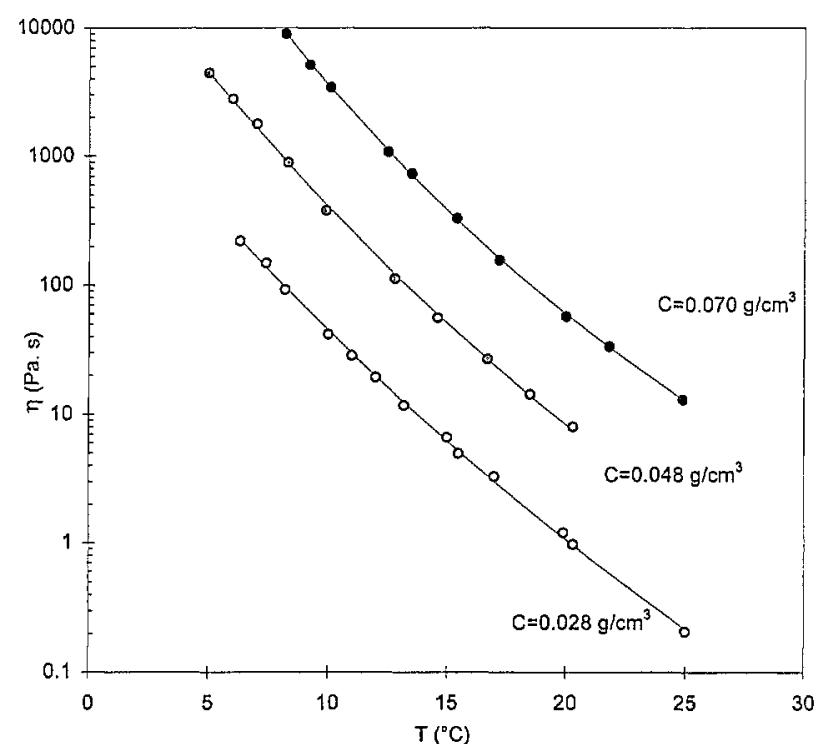

Figure 2. Temperature dependence of the zero shear viscosity $\eta$ for three different concentrations. The experimental data are well fitted by a Vogel-Fulcher law that is suggestive of liquids near the glass transition.

typical temperature dependence of the viscosity for three different concentrations. All attempts to fit the temperature behavior of the viscosity using power laws, as expected for gelation, legd to very high and unrealistic exponent values. The experimental data are well fitted by a Vogel-Fulcher law:

$$
\eta=\eta_{0} \mathrm{e}^{E /\left(T-\mathrm{T}_{\mathrm{g}}\right)}
$$

where $\eta_{0}, E$, and $T_{\mathrm{g}}$ are adjustable parameters that depend on the sample concentration. Such temperature behavior is suggestive of liquids near the glass transition. For the different concentrations studied, the determined temperatures $T_{\mathrm{g}}$ are compatible with the glass transition temperatures of polystyrene taking into account the plasticizer effect of $n$-heptane. ${ }^{9}$ However, such a determination of the glass transition temperature is not sufficiently accurate to be reported in this paper. Further experiments are planned using an NMR technique which will allow us to probe the local dynamics of polystyrene.

As the temperature decreases, both the zero shear viscosity, $\eta$, and the longest relaxation time, $\tau_{\mathrm{R}}$, increase by the same factor, leading to a plateau modulus $G=$ $\eta / \tau_{\mathrm{R}}$ independent of the temperature within the explored temperature range (Figure 3 ). The plateau modulus varies linearly with the concentration $C$ (Figure 4 ):

$$
G(\mathrm{~Pa})=(1.30 \pm 0.07) \times 10^{4} \mathrm{C}-(150 \pm 50)
$$

where $C$ is expressed in grams per cubic centimeter. Note that relation 2 leads to a zero plateau modulus for $C=1.2 \times 10^{-2} \mathrm{~g} / \mathrm{cm}^{3}$, in agreement with the value of $C^{*}$ previously reported. This behavior has to be compared to the reptation regime of homopolymer semidilute solutions in which $G$ varies as $C^{2.3}$. Indeed, in spite of a concentration higher than $C^{*}\left(1<C / C^{*}<\right.$ 8 ), copolymers studied here are never entangled. This is due to the $n_{\mathrm{e}}$ blobs needed for polymers to be entangled. The number $n_{\mathrm{e}}$ is equal to $180 \pm 50$ and 110 \pm 10 for polystyrene and polyisoprene, respectively. ${ }^{10,11}$ This leads to an entanglement regime at concentrations higher than $C / C^{*}=10$ for homopolystyrene and $C / C^{*}$ $=7$ for homopolyisoprene. Thus, entanglement effects cannot be involved in the explanation of the concentration dependence of the elastic modulus. Let us consider 


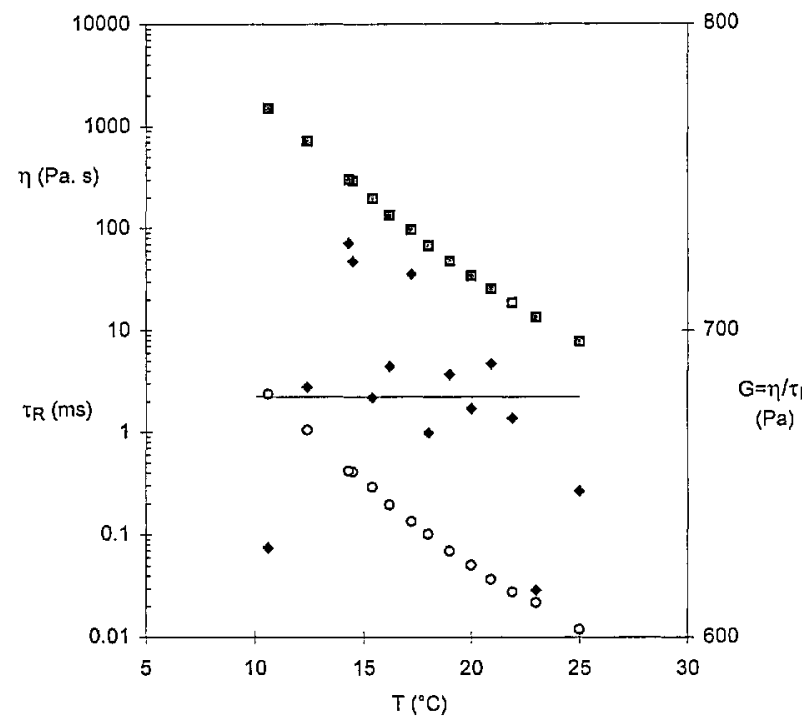

Figure 3. Temperature dependence of the zero shear viscosity $\eta$ (log scale, squares), of the longest relaxation time $\tau_{\mathrm{R}}$ (log scale, circles), and of the plateau shear elastic modulus $G=$ $\eta / \tau_{\mathrm{R}}$ (linear scale, diamond) for a given concentration $C=0.064$ $\mathrm{g} / \mathrm{cm}^{3}$.

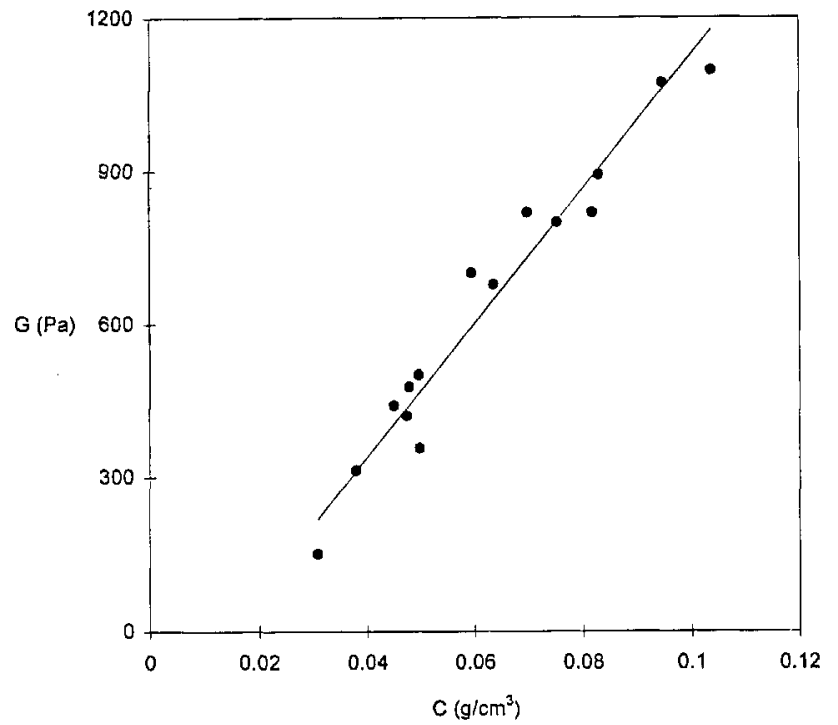

Figure 4. Concentration dependence of the plateau shear elastic modulus $G$. A linear behavior is found in agreement with a Rouse modulus $k T C / M$.

the case of the Rouse semidilute regime or gels made of end-linked and unentangled polymers at the swelling equilibrium. Then, the modulus is due to the elasticity of the entire chain made of $N / g$ blobs of size $\xi$ ( $N$ being the degree of polymerization and $g$ the number of monomers per blob). The elastic modulus in this regime is thus equal to $G_{\text {Rouse }}=k T /\left((N / g) \xi^{3}\right)=\mathcal{N} k T(C / M)$, where $\mathcal{F}$ is the Avogadro number. The mass of the triblock copolymer leads to $G_{\text {Rouse }}(\mathrm{Pa})=1.5 \times 10^{4} C$, which is in good agreement with the concentration behavior of the measured elastic modulus reported in relation 2 . Thus, in a selective solvent we measure an elastic modulus that is comparable to the one for a semidilute solution (Rouse regime) in a good solvent or to the one for a gel made of end-linked copolymers at the swelling equilibrium. In other words, the elastic modulus corresponds to chains pulled by their ends. Here, it is important to underline that this concentration behavior is obtained as soon as the overlap concentration of the copolymer is reached. That means that the system we have

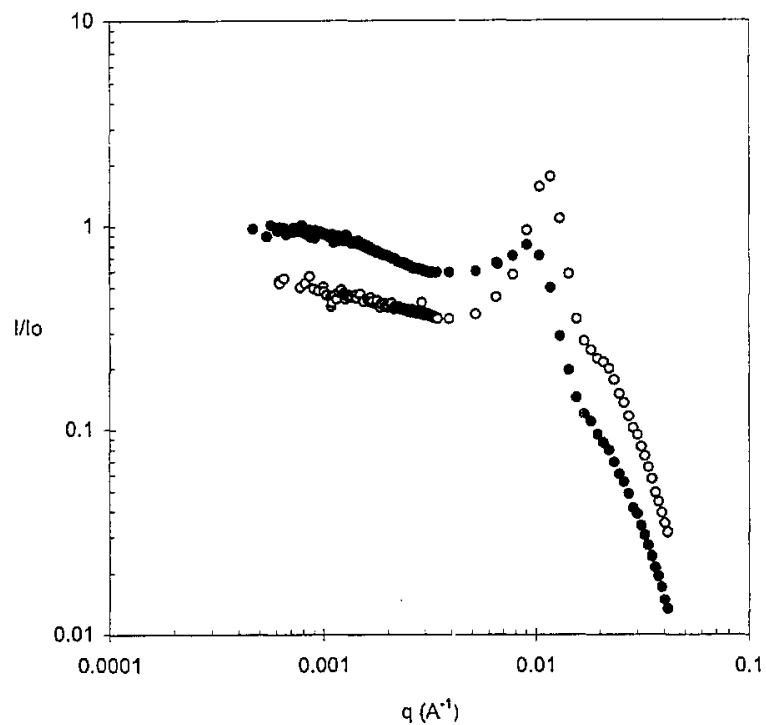

Figure 5. Reduced scattering function, $I / I_{0}=(I(q) / C) /(I /$ $C)_{q \rightarrow 0, C \rightarrow 0}$ measured by light scatteririg and small-angle neutron scattering, as a function of the iransfer vector $q$ for two concentrations taken as examples. In this representation, neutron scattering results are scaled by an appropriate factor in order to coincide with the high $q$ range of the light scattering data. Open and filled symbols cor:espond to $C=0.04 \mathrm{~g} / \mathrm{cm}^{3}$ and $C=0.027 \mathrm{~g} / \mathrm{cm}^{3}$, respectively.

studied in this paper does not come under the theoretical approach recently developid by Semenov et al. ${ }^{12}$ These authors consider the semidilute solution of "flower"-like micelles. In their joicture, the flowers hold more and more hands with i, heir neighbors as the concentration increases. The elastic modulus being proportional to the number of efficient elastic chains per volume unit, such behavior would lead to $G \infty C^{x>1}$. This discrepancy between the theoretical behavior and the system we have studied has to be compared with the discrepancy that is also observed in the dilute regime.

In addition to the concentration behavior of the modulus, one has to account for the drastic temperature dependence of the zero shear viscosity and of the viscoelastic relaxation time which seem to be controlled by the polystyrene glass transition. The simplest structure that is able to account for the concentration dependence of the elasticity and for the temperature dependence of the viscosity consists of a transient network of polystyrene nodes linked together by polyisoprene middle blocks. Scattering experiments were performed in order to determine this structure.

\section{Light and Small-Angle Neutron Scattering}

In order to investigate the scattering function $I(q)$ over a wide $q$ range, neutron and light scattering experiments were performed. In Figure 5 the whole reduced scattering function, $I / I_{0}=(I(q) / C) /(I / C)_{q \rightarrow 0, C \rightarrow 0}$, is plotted as a function of $q$ for two concentrations taken as example. In this representation, neutron scattering results are scaled by an appropriate factor which depends on the concentration in order to coincide with the high $q$ range of the light s.cattering data. Strictly speaking, such superposition of neutron and light scattering data is only valid for homopolymers. In the case of copolymers, the contrast length ratio of the two monomer species is different for light and neutron experiments and consequently it is not exactly the same object that is viewed by thesie two techniques. The scattering function shows two features: a well-marked peak, characteristic of ordered structures, and at low $q$ 


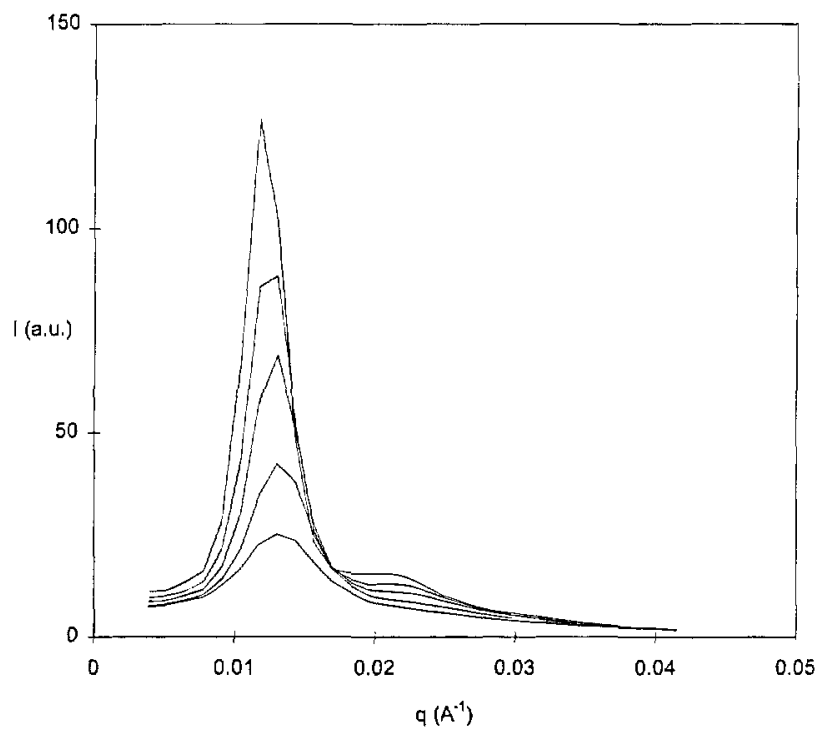

Figure 6. Small-angle neutron-scattered intensity measured for a given concentration $\left(C=0.063 \mathrm{~g} / \mathrm{cm}^{3}\right)$ at different temperatures (from the upper curve to the lower curve, $T=$ $10,20,30,40$ and $50^{\circ} \mathrm{C}$ ).

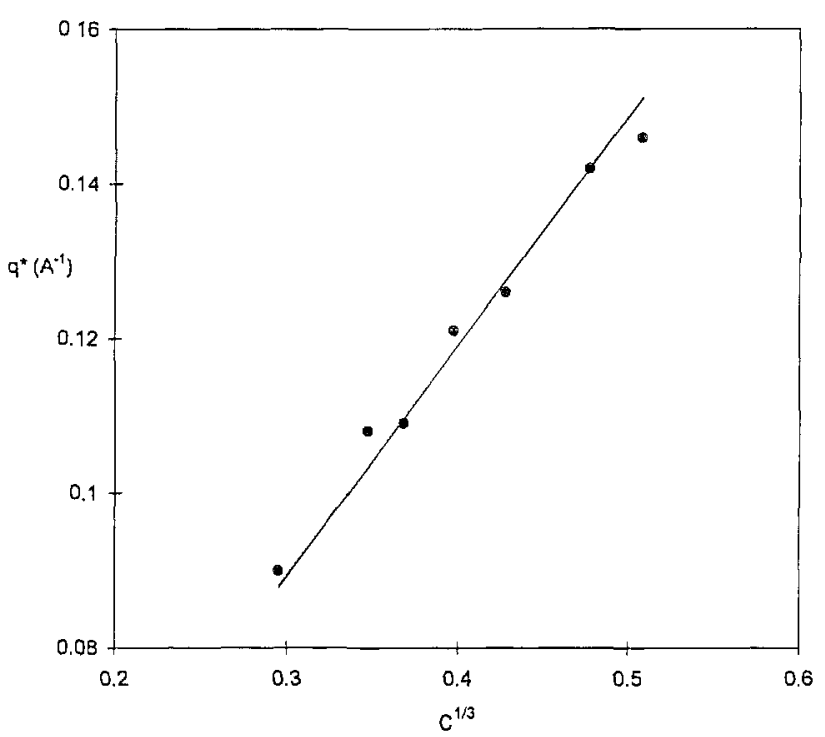

Figure 7. Location $q^{*}$ of the scattered intensity peak as a function of $C^{1 / 3}$, where $C$ is the concentration expressed in grams per cubic centimeter. The linear behavior is characteristic of a three-dimensional structure.

an increase of scattered intensity with decreasing $q$, typical of disordered and compressible structures. These two features will be now discussed.

Small-angle neutron scattering experiments were performed using natural contrast and deuterated $n$ heptane. Whatever the concentration and the temperature, neutron-scattered intensity, $I$, as a function of the transfer vector, $q$, exhibits an intense peak. The width of this peak is of the order of the spectrometer resolution. Within the accuracy allowed by the small number of points defining the peak, its position $q^{*}$ is independent of the temperature for a given concentration (Figure 6). In Figure 7 the variation of $q^{*}$, proportional to the inverse of the lattice unit cell dimension, is shown as a function of $C^{1 / 3}$; a linear behavior is found:

$$
q^{*}\left(\mathrm{~nm}^{-1}\right)=(0.297 \pm 0.003) C^{1 / 3}
$$

$C$ being expressed in grams per cubic centimeter. This result constitutes a major piece of evidence for a threedimensional structure. Actually, this peak presents a

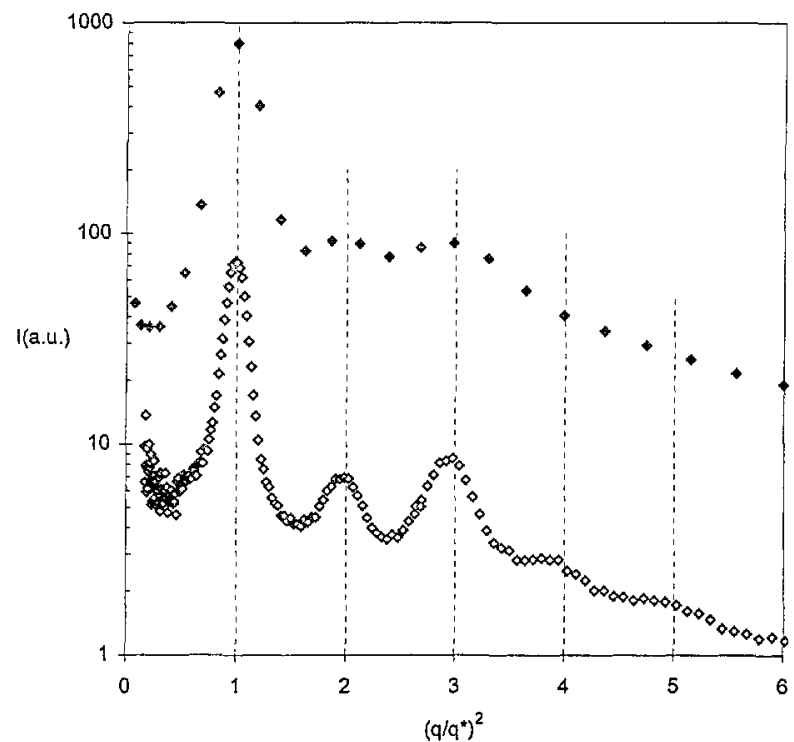

Figure 8. Small-angle neutron-scattered intensity as a function of $\left(q / q^{*}\right)^{2}$ with $q^{*}=1.7 \times 10^{-2} \AA^{-1}$, measured at $20^{\circ} \mathrm{C}$ for a given concentration $C=0.16 \mathrm{~g} / \mathrm{cm}^{3}$. Upper curve, filled symbols: static measurement performed on Pace at Saclay $(\lambda$ $=10 \AA, D=3.2 \mathrm{~m}$ ). Lower curve, open symbols: measurement performed on the same spectrometer using the time of flight technique $(D=4.5, \Delta \lambda / \lambda=1 \%)$ and regrouping all the detector data by $\Delta q / q=1 \%$. The upper curve has been multiplied by an arbitrary factor for clarity.

shoulder at higher $q$ values that is more and more pronounced as the temperature decreases. In fact, for concentrations higher than $7 \times 10^{-2} \mathrm{~g} / \mathrm{cm}^{3}$ and at temperatures lower than $30{ }^{\circ} \mathrm{C}$, neutron scattering spectra show two smaller peaks, the positions of which are $(2)^{1 / 2} q^{*}$ and $(3)^{1 / 2} q^{*}$, respectively (Figure 8 ). In order to confirm this result, small-angle neutron scattering measurements were performed using the time-of-flight technique $^{13}$ on one sample. In Figure 8 , the scattered intensity is plotted as a function of the reduced square transfer vector $\left(q / q^{*}\right)^{2}$. With this resolution, up to five Bragg peaks are displayed. Their respective positions reveal unambiguously a cubic lattice structure, without any possibility to decide between simple cubic or bodycentered cubic structure. One has to recall that lamellar phases should give second and third peaks at $2 q^{*}$ and $3 q^{*}$, respectively, ${ }^{14}$ while disordered lamellar phases should give only one peak. ${ }^{15}$

The lattice unit cell dimension $d$ can be deduced from the position $q^{*}$ of the first scattered intensity peak. Assuming a body-centered cubic structure, one has $d=$ $2(2)^{1 / 2} \pi / q^{*}$. Using relation 3 for the concentration dependence of $q^{*}, d$ varies between 60 and $100 \mathrm{~nm}$ and is found to be

$$
d(\mathrm{~nm})=(30.0 \pm 0.3) C^{-1 / 3}
$$

Note that $60 \mathrm{~nm}$ corresponds to twice the hydrodynamic radius of the diblock copolymer micelles mentioned in section 1. Another way to present the same result is to consider the number $f$ of polymer chains that contribute to form a polystyrene node. In the case of a bodycentered cubic structure, there are 2 nodes per lattice unit cell. Thus, basically one gets

$$
f=\frac{C \mathcal{M}}{M} \frac{d^{3}}{2}
$$

The number $f$, which corresponds to the number of copolymer chains per node, is found to be independent of the concentration and equal to 


$$
f=48 \pm 2
$$

This indicates that decreasing the concentration acts as a simple dilation of the lattice, the structure remaining unaffected. It is important to compare the number of copolymer chains per node with the association number measured in dilute solution. In a previous paper ${ }^{4}$ it was shown that the aggregates, studied at temperatures above $30^{\circ} \mathrm{C}$ in dilute solution, have a loose structure, i.e., with an internal concentration $C^{*}$ of the order of that of the unimer. Such "animals" have a small association number that increases with increasing concentration, the maximum value being equal to 8 . On the other hand, diblock copolymers PS-PI, corresponding to half of the triblocks here studied, form spherical and starlike micelles having an association number just equal to the number $f$. The difference between triblock and diblock aggregates was imputed to the loss of entropy due to the backfolding of the middle block which prevents telechelic triblock to form flowerlike micelles. ${ }^{16,17}$ Relation 6 shows that such a difference between triblock and diblock copolymer association number vanishes in the semidilute regime. This can be explained because the two terminal blocks of a given copolymer can belong to two neighboring micelles, and no loops are needed in semidilute solutions. In addition, the association number being identical, it is interesting to compare the first neighbor distance $d(3)^{1 / 2} / 2$ of the triblock lattice to twice the hydrodynamic radius $2 R_{\mathrm{h}}$ $=58 \mathrm{~nm}$ of the diblock micelles. A starlike structure involves a stretching of the arms; however, the above comparison shows that, for the lowest concentrations, triblock copolymer chains are stretched even more.

Now, let us come back to the elastic modulus expression. The shear elastic modulus was found to correspond to a Rouse modulus equal to $k T /(C / M)$. Thus, using eq 5 , one can obtains the modulus as a function of the lattice unit cell dimension $d$ and the number $f$ of chains per node, which corresponds to the number of cross-links per volume unit:

$$
G=\frac{2 f(k T)}{d^{3}}
$$

The SANS time-of-flight technique allows the spectrum to be studied as a function of the transfer vector resolution. This can be done by considering the width $\Gamma$ of the first Bragg peak measured on each detector, each of them having a given angular resolution $\Delta \theta / \theta$. In Figure 9, the relative width $\Gamma / q^{*}$ of the first peak is plotted as a function of the resolution $\Delta q / q$. The linear behavior passing through the origin shows that the width of the peak is entirely due to the spectrometer resolution. Consequently, these experiments do not allow one to deduce the size $\sigma$ of the crystalline domain. However, assuming for the Bragg peak a Lorentzian form $\left(\left(q-q^{*}\right)^{2}+1 / \sigma^{2}\right)$, the best spectrometer resolution $\left(\Gamma / q^{*}\right)_{\min }=4 \%$ allows us to determine a lower limit for $\sigma$. Thus, $1 /\left(q^{*} \sigma\right) \ll\left(\Gamma / q^{*}\right)_{\min }=0.04$ and the expression of the lattice unit cell dimension $d=2(2)^{1 / 2} \pi / q^{*}$ lead to

$$
\sigma / d>3
$$

which means that at least 27 unit cells contribute to form a crystalline domain.

In the $q$ range of light scattering, the scattered intensity is independent of the temperature. The concentration dependence is shown in Figure 1, in which the reduced scattered intensity per monomer, $(I / C)_{q \rightarrow 0}$ $(I / C)_{q \rightarrow 0, C \rightarrow 0}$ measured in $n$-heptane is compared to the one measured in THF. In good solvent (THF), results

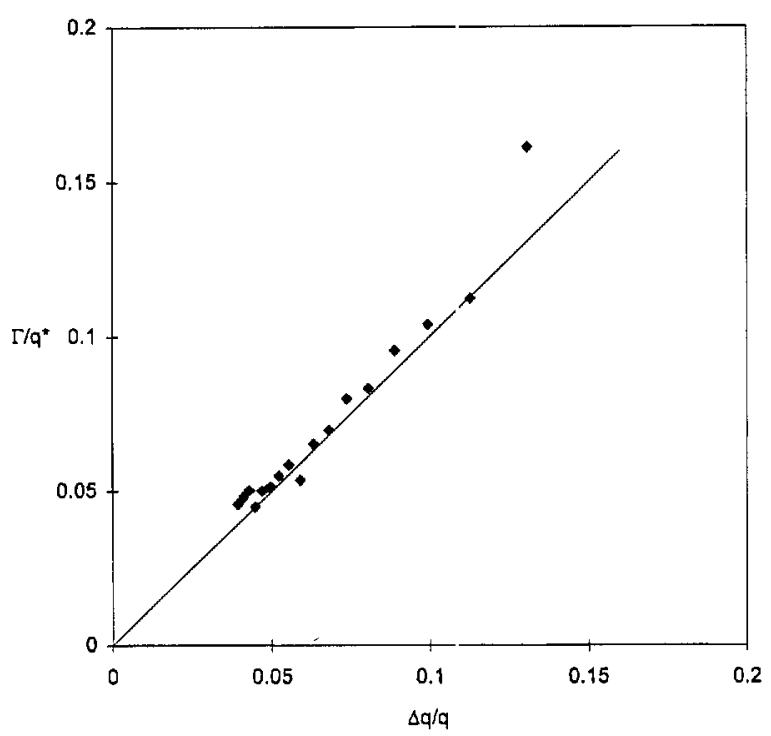

Figure 9. Relative width $\Gamma / q^{*}$ of the first Bragg peak as a function of the resolution $\Delta q / q$. With $\Delta q / q=\Delta \theta / \theta+\Delta \alpha / \alpha+$ $\Delta \lambda / \lambda$; angular resolution $\Delta \theta / \theta=0 . E /(d+2)$ with $d$ the beam detector distance (from 1 to 30 ); collimation resolution calculated following ref 30 ; wavelength resolution $\Delta \lambda / \lambda=1 \%$. The line is a guide for the eyes having a slope of 1 and passing through the origin.

are compatible with the scaling; behavior expected for linear homopolymers for which the scattered intensity per monomer is expected to clecrease as $(I / C)_{q \rightarrow 0} /(I /$ $C)_{q \rightarrow 0, C \rightarrow 0}=(k T / M)(\partial \pi / \partial \mathrm{C})^{-1}=\left(C^{\prime} / C^{*}\right)^{-1.31}$. In $n$-heptane for $C / C^{*}>0.1$, as mentioned above, the increase of the reduced scattered intensity per monomer reveals the growth of triblock aggregates. Around $C / C^{*}=1$, the scattered intensity per monomer' starts to decrease with increasing concentration, as expected for semidilute solutions, because of the screening of concentration fluctuations. Nevertheless, even for the highest concentration studied here, the scattered intensity remains higher by more than one decade than the corresponding intensity expected in good solver.t. Because of its strong concentration dependence, the light-scattered intensity per monomer is mostly due to polystyrene nodes constituting the bcc structure rather than due to a deficiency of the screening as for starlike polymer. ${ }^{18}$ In addition, one has to note that in the case of star polymers the scattered intensity increases with $q$ for $q$ $\rightarrow 0$. Another difference between star polymer ordering and our results for triblock copilymers is that for star polymers the scattered intensity at $q^{*}$ is never higher than $(I / C)_{q \rightarrow 0, C \rightarrow 0}$. This later behavior is characteristic of hard-sphere liquid ordering. As shown in Figure 5, this is never the case for triblock copolymers that undergo crystal ordering.

\section{Quasi-Elastic Light Scattering}

A quasi-elastic light scattering device allows the timedependent fluctuations of the refractive index to be analyzed by computing the autocorrelation function of the scattered intensity $I(t)$. The time-dependent dynamical structure factor $S(q, t)$ is equal to

$$
S(q, t)=\frac{\langle\delta I(0) \delta I(t)\rangle^{2}}{\left\langle\delta I(t)^{2}\right\rangle}
$$

However, in order to clearly outline what is measured, it is more convenient to discuss in the frequency domain. The dynamical structure factor $S(q, \omega)$ is proportional to the imaginary part of the dynamical longitudinal 


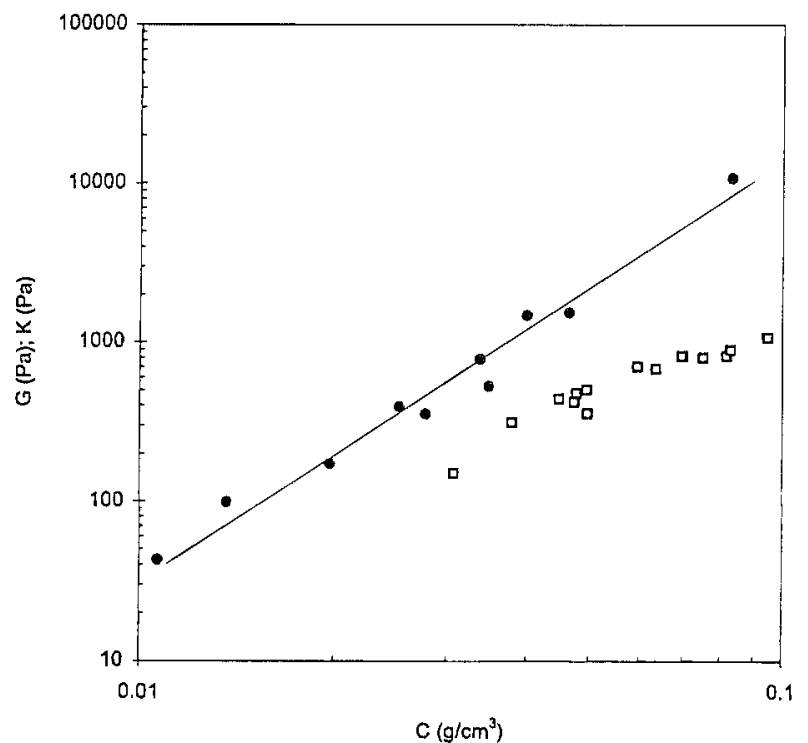

Figure 10. Bulk osmotic modulús $K$ (filled symbols) and shear elastic plateau modulus $G$ (open symbols) as a function of the concentration $C$. Depending on the concentration $K$ is always greater than $G$ by a factor between 3 and 10 . The straight line corresponds to the best fit: $K_{0}(\mathrm{~Pa})=(6 \pm 0.6) \times$ $10^{6} \mathrm{C}^{(2.65 \pm 0.05)}$

compliance $J=1 / M$, where $M$ is the longitudinal elastic modulus: ${ }^{19,20}$

$$
S(q, \omega)=\frac{n k T}{\omega} \operatorname{Im}\{1 / M(\omega)\}
$$

with $n$ the number of particles per volume unit. Neglecting density fluctuations compared to concentration fluctuations, the longitudinal elastic modulus is a sum of the bulk osmotic modulus $K$ and of the shear elastic modulus $G$ : $M(\omega)=K(\omega)+4 / 3 G(\omega)$. Let us evaluate the respective contribution of the bulk and shear moduli to the dynamical structure factor. As copolymer solutions are liquid in the temperature range investigated, there is no shear contribution to the static light scattering measurements (zero frequency). Thus, the total scattered intensity per monomer extrapolated to zero transfer vector leads to the zero-frequency bulk osmotic modulus $K_{0}$ :

$$
(I / C)_{q \rightarrow 0} \propto \frac{k T C}{K_{0}}
$$

A temperature-independent behavior is found. In Figure 10 , the bulk osmotic modulus $K_{0}$ and the shear elastic plateau modulus $G$ are plotted as a function of the concentration. One can see that $K_{0}$ is always higher than $G$ by a factor between 3 and 10, depending on the concentration. Let us assume the simplest forms for the expressions of the two moduli $\left(K^{*}=K_{0}(1+i \omega \tau)\right.$ and $\left.G^{*}=G i \omega \tau_{\mathrm{R}} /\left(1+i \omega \tau_{\mathrm{R}}\right)\right)$. In addition, we will see later on that the long time cutoff of the quasi-elastic relaxation function is always higher than the shear elastic relaxation time, $\tau_{\mathrm{R}}$, by more than two decades (Figure 14, which will be discussed later on). Consequently, the bulk osmotic modulus is always higher than the shear elastic modulus whatever the frequency. Thus, quasielastic light scattering experiments are mainly sensitive to osmotic relaxation $(K)$ rather than shear relaxation $(G)$. Here it is important to note that, as light scattered intensity is mostly due to polystyrene nodes constituting the bcc structure, quasi-elastic light scattering experiments are sensitive to the relaxation of the concentration fluctuations of these nodes.

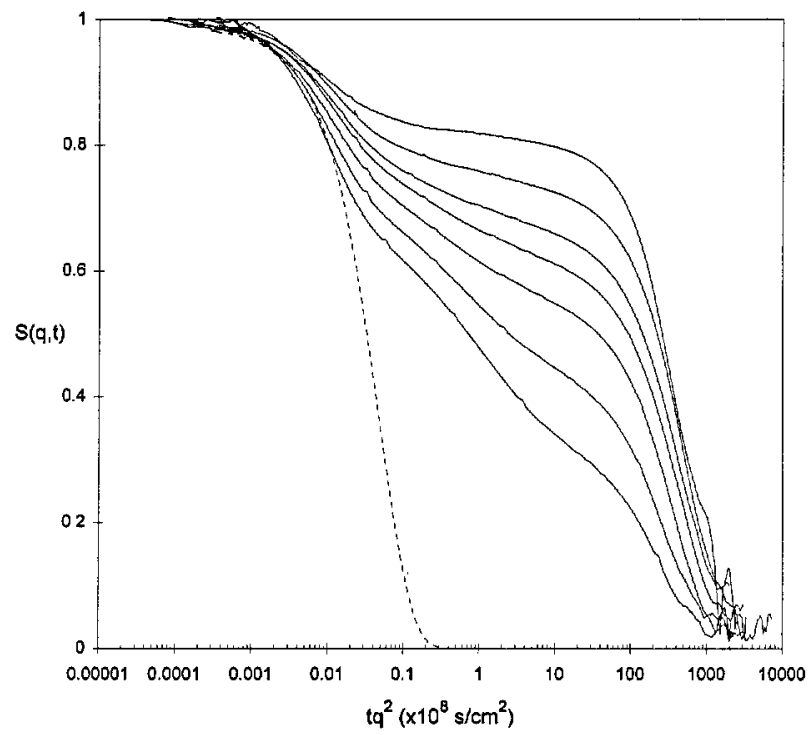

Figure 11. Dynamic structure factor $S(q, t)$ measured by quasi-elastic light scattering at a given temperature $T=30$ ${ }^{\circ} \mathrm{C}$ and a given concentration $\mathrm{C}=0.068 \mathrm{~g} / \mathrm{cm}^{3}$. The different curves correspond to different transfer vectors $q$ : from the upper to the lower curve $q / q^{*}=0.11,0.16,0.22,0.27,0.32$, 0.45 , and 0.60 . The dotted line is a guide for the eyes corresponding to a simple-exponential decay.

The relaxation function extends over seven decades and cannot be accounted for by either a single-exponential or a stretched exponential relaxation. In Figure 11 , typical dynamical structure factors obtained at different $q$ values for a given temperature and a given concentration are plotted as a function of $t q^{2}$ and compared to a single-exponential decay. Note that in such a representation all the curves have to be superimposed if the relaxation process corresponds to diffusive motions. As one can see in Figure 11, this is the case for the short-time and long-time parts of the relaxation curves but not at all for the intermediatetime scale. These three time regimes will be discussed later, but let us already remark that such a $q$ dependence of the dynamical structure factor is characteristic of anomalous slow dynamics. To outline this feature, one has to keep in mind that quasi-elastic measurements performed at low $q$, i.e., large length scale, are equivalent to probing the long-time behavior of the material. In Figure 11, it appears clearly that the lower $q$ the slower the relaxation. This corresponds to an effective diffusion coefficient decreasing with increasing time.

Figure 12 and Figure 13 illustrate the influence on the dynamical structure factor of the temperature and of the concentration, respectively. One can see that at short time the dynamical structure factor seems to be independent of these macroscopic variables while at long time it depends strongly on them.

As mentioned above, the dynamical structure factor shows a short-time diffusive process. The corresponding diffusion coefficient $D_{1}$, determined by the initial slope of $\ln (S(q, t))$ vs time, is found to be independent of the temperature and of the concentration:

$$
D_{1}=(28 \pm 5) \times 10^{-8} \mathrm{~cm}^{2} / \mathrm{s}
$$

Such behavior reveals that the local properties, probed by the short-time dynamical structure factor, are independent of the macroscopic variables $(T$ and $C$ ) within experimental accuracy.

In contrast, the long-time diffusive process is found to be strongly affected by the macroscopic properties of 


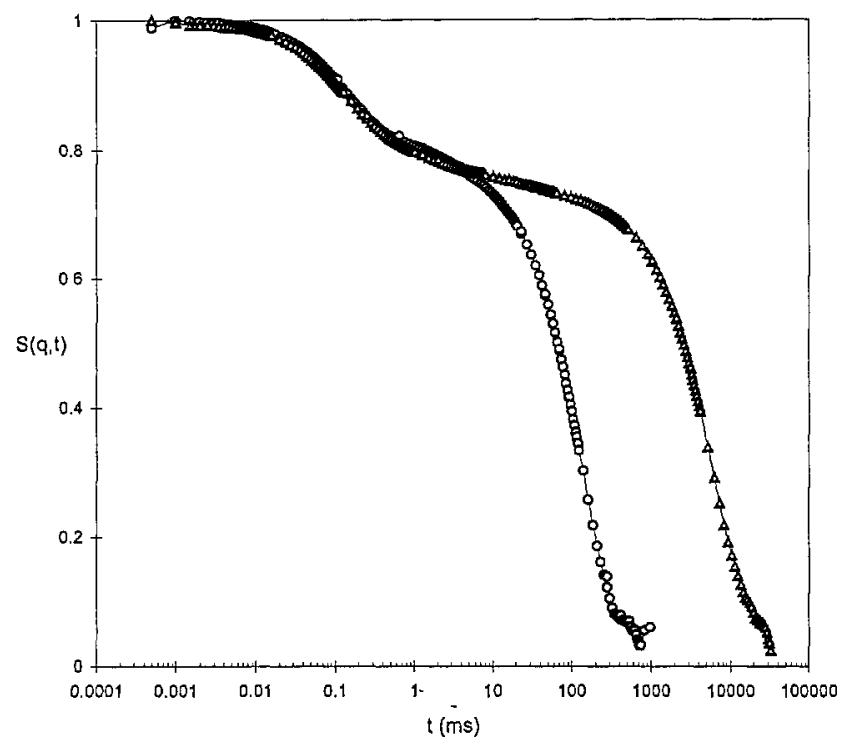

Figure 12. Dynamic structure factor $S(q, t)$ measured by quasi-elastic light scattering at a given concentration $(C=$ $\left.0.068 \mathrm{~g} / \mathrm{cm}^{3}\right)$, for a given scattering angle $\left(q / q^{*}=0.16\right)$ and two different temperatures (upper curve, triangles $T=30^{\circ} \mathrm{C}$; lower curve, circles $T=50^{\circ} \mathrm{C}$ ).

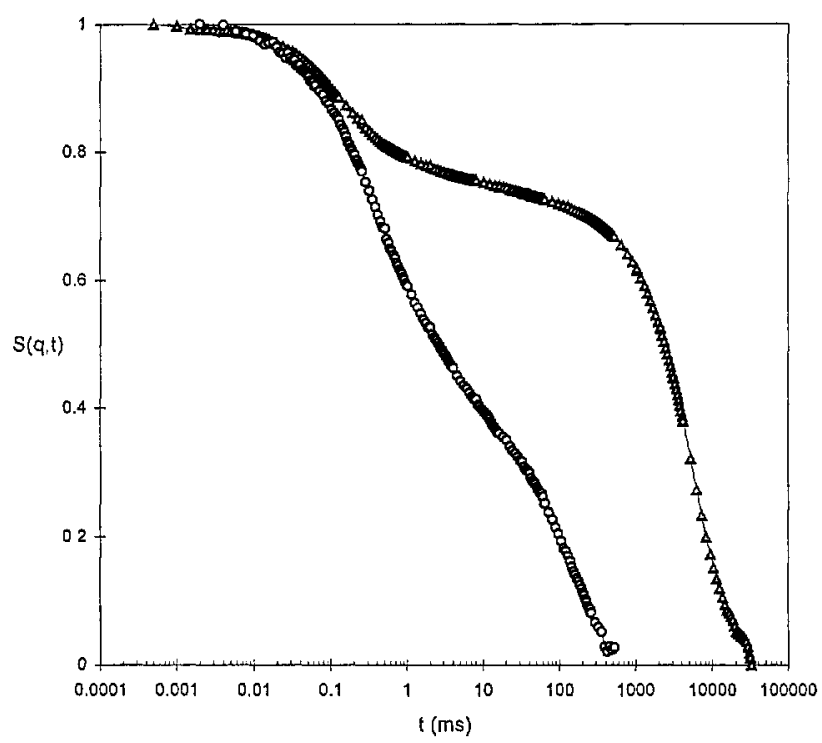

Figure 13. Dynamic structure factor $S(q, t)$ measured by quasi-elastic light scattering at a given temperature $(T=30$ $\left.{ }^{\circ} \mathrm{C}\right)$, for a given scattering angle $\left(q=9.25 \times 10^{-3} \mathrm{~nm}^{-1}\right)$ and two different concentrations (upper curve, triangles $C=0.068$ $\mathrm{g} / \mathrm{cm}^{3}$; lower curve, circles $C=0.034 \mathrm{~g} / \mathrm{cm}^{3}$ ).

the solution. The long-time diffusion coefficient $D_{3}$ is determined by the slope of $\ln (S(q, t))$ vs time in the longtime part of the correlation function. The diffusion coefficient $D_{3}$ depends on both concentration and temperature. In order to quantify this dependence, it is worthwhile to compare the long-time diffusion coefficient $D_{3}$ to the ratio $d^{2} / \tau_{\mathrm{R}}$, which has the dimension of a diffusion coefficient, $d$ being the lattice unit cell dimension and $\tau_{\mathrm{R}}$ the rheological relaxation time. In Figure 14, the diffusion coefficient $D_{3}$, measured at different temperatures and concentrations, is plotted as a function of $d^{2} \tau_{\mathrm{R}}$. One can see that these two quantities are proportional; one finds

$$
\frac{D_{3}}{d^{2} / \tau_{\mathrm{R}}}=(5.5 \pm 0.5) \times 10^{-3}
$$

for two decades variation of $d^{2} / \tau_{R}$. This result indicates

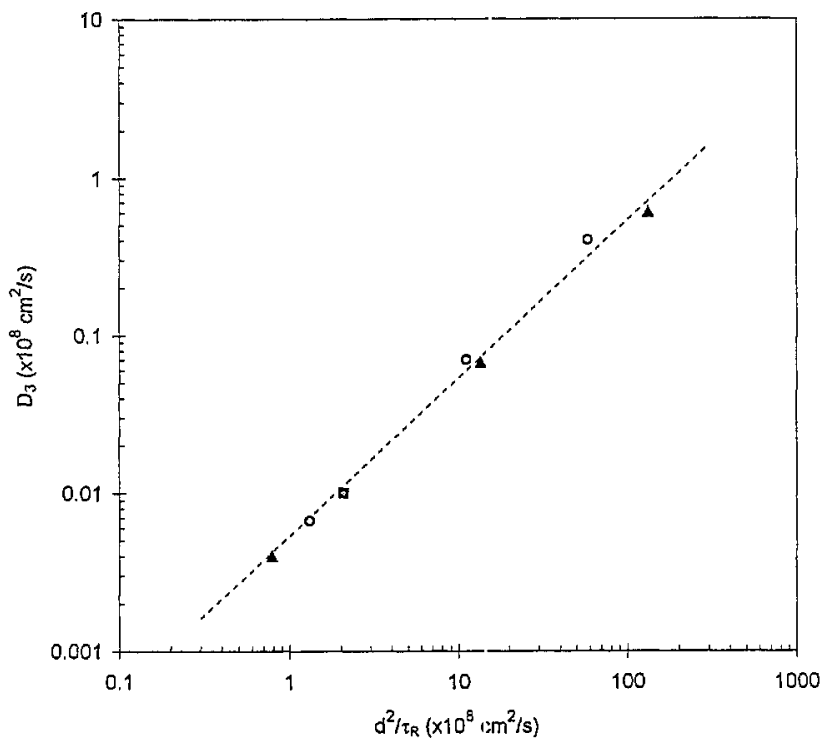

Figure 14. Long-time diffusion coefficient $D_{3}$ as a function of $d^{2} / \tau_{\mathrm{R}}, d$ being the lattice unit cell dimension measured by small-angle neutron scattering and $\tau_{R}$ being the longest rheological relaxation time measured with the magnetorheometer. From right to left: triangles $C=0.034 \mathrm{~g} / \mathrm{cm}^{3}, T=40$, 30 , and $20^{\circ} \mathrm{C}$; square $C=0.055 \mathrm{~g} / \mathrm{cm}^{3}, T=30^{\circ} \mathrm{C}$; circles $C=$ $0.068 \mathrm{~g} / \mathrm{cm}^{3}, T=50,40$, and $30^{\circ} \mathrm{C}$.

that it is possible to account for the long-time behavior of the quasielastic relaxation curves using the reduced time scale $t(q d)^{2} / \tau_{\mathrm{R}}$.

At the intermediate-time scale, the dynamical structure factor is typical of slow dynamics, with a relaxation plateau that is more and mcre pronounced as the temperature decreases and as the concentration increases. Actually, this behavior as well as the overall shape of the relaxation curves is quite similar to the dynamical structure factor experimentally observed for colloids near the glass transition ${ }^{21,22}$ or expected for supercooled liquids. ${ }^{23,24}$ Starting from this analogy, the following model is proposed in order to account for the three regimes mentioned above:.

1. The short-time, diffusive regime is imputed to the motion of each polystyrene node around its quasiequilibrium crystalline position. This diffusive motion then saturates after a certain time $t_{1}$, reflecting the fact that each node is in a potential well created by its surrounding, which acts as a cage. This accounts for the shoulder in the dynamical structure factor, which levels off at a plateau value $f_{\mathrm{q}}$.

2. This plateau would extend to infinite times if the system were in a solid state. However, one has to keep in mind that the system is paradoxically a liquid. That means that the local neighborhood of one specific PS node changes with time. In othir words, cages fluctuate leading to an anomalous collective diffusion of the nodes. This can be conceived as a succession of jumps between $\operatorname{traps}^{25-27}$ with a broad distribution of trapping times $\tau$, decaying as $\tau^{-(1+\beta)}$, and givıs rise to the stretched exponential $\beta$ decay of $S(q, t)$ as $f_{\mathrm{q}} \exp \left[-\left(t / t_{2}\right)^{\beta}\right]$. Such a $\beta$ decay can be derived by assuning that the elementary events that contribute to this cooperative diffusion correspond to jumps from one fotential hole to another. An exponential distribution of the energy barrier $\Delta E$ as $P(\Delta E)=\mathrm{e}^{-\Delta E / E_{0} / E_{0}}$, leads to a trapping time distribution $P(\tau) \sim \tau^{-\langle 1+\beta)}$, with $\beta=k T / E_{0}<1$ and $E_{0}$ the characteristic energy of the distribution.

3. As mentioned above (eq 13), the terminal region of $S(q, t)$ reveals the liquid feature of the system. This is due to a long-time cutoff, $i, 3$, of the trapping time 
distribution function. For time longer than $t_{3}$, the anomalous diffusion vanishes and diffusive relaxatior. takes place again. The time $t_{3}$ can be due to the cutof: of the distribution of the number $f$ of polystyrene blocks. that contribute to form a node. This distribution has a. large number cutoff because the concentration in a node. is a function of the association number $f$ and cannot be larger than 1. In this picture, time $t_{3}$ is related to the maximum lifetime of a node, i.e., a transient cross-link. and consequently to the longest relaxation time $\tau_{\mathrm{F}}$. measured by rheology.

In order to rescale the dynamical structure factor measured at different scattering angles, it was assumed that a single, time-dependent length scale $r(t)$ is needed to obtain a "master curve" for $S(q, t)$ as a function of" $q^{2}\left\langle r(t)^{2}\right\rangle$. This length scale, which corresponds in fact to a mean square displacement, has to account for the three regimes discussed above. Its simplest form is

$$
\left\langle r(t)^{2}\right\rangle=L^{2}\left[1-\exp \left(-\frac{t}{t_{1}}\right)+\left(\frac{t}{t_{2}}\right)^{\beta}+\left(\frac{t}{t_{3}}\right)\right]
$$

where $L^{2}$ is the mean square displacement at which the local diffusion starts to saturate. The short-time and long-time diffusion coefficients being independently determined, only three parameters are independent in eq 14; actually, one has $L^{2}=D_{1} t_{1}$ and $t_{3}=L^{2} / D_{3}$. For a given temperature and a given concentration, it is possible to optimize the three parameters $t_{1}, t_{2}$, and $\beta$ in order to account for the $q$ dependence of the dynamical structure factor. The master curve so obtained as well as the exponent $\beta$ (Figure 15) is found to be independent of the temperature. For comparison, the $q$-dependent dynamical structure factors measured at the same concentration and three different temperatures are plotted as a function of $t(q d)^{2}$ (Figure 15a) and as a function of $q^{2}\left\langle r(t)^{2}\right\rangle$ (Figure $15 \mathrm{~b}$ ). The fact that the master curve is found to be independent of the temperature indicates that the cage size $L$ is also independent of the temperature. This can be seen qualitatively in Figure 12, which clearly shows that the relaxation plateau appears at a time $t_{1}$ independent of the temperature. Using eq 13 and the above definition of $t_{3}$, one can write the following relation between $t_{3}$ and $\tau_{\mathrm{R}}$ :

$$
t_{3}=200(L / d)^{2} \tau_{\mathrm{R}}
$$

However, the master curves still remain dependent on the concentration. In Figure 16, one can compare the dynamical structure factor vs $q^{2}\left\langle r(t)^{2}\right\rangle$ for two concentrations $\left(C_{1}=0.034 \mathrm{~g} / \mathrm{cm}^{3}\right.$ and $\left.C_{2}=0.068 \mathrm{~g} / \mathrm{cm}^{3}\right)$, for which $\beta=1$ and $\beta=0.4$, respectively. It appears that the curves are superimposed at short distances but are separated by one decade at long distances. In order to outline this concentration dependence, it is worthwhile to compare the lattice unit cell dimension $d$ to the cage size $L$. Let us recall that these two quantities were independently determined: the first by small-angle neutron scattering and the second through the product $D_{1} t_{1}$. The time $t_{1}$, independent of the temperature, is determined by the scaling of $S(q, t)$ vs $q^{2}\left\langle r(t)^{2}\right\rangle$; it corresponds roughly to the beginning of the relaxation plateau. The corresponding diffusion coefficient $D_{\mathbf{1}}$, independent of temperature and concentration, is determined by the initial slope of the dynamical structure factor. For the two concentrations analyzed, it is found that $L / d \cong 0.7$ for $C=0.068 \mathrm{~g} / \mathrm{cm}^{3}$ and $L / d \cong 3$ for $C=$ $0.034 \mathrm{~g} / \mathrm{cm}^{3}$. This indicates that the lower the concentration the smaller the cage effect. This result is qualitatively in agreement with static properties
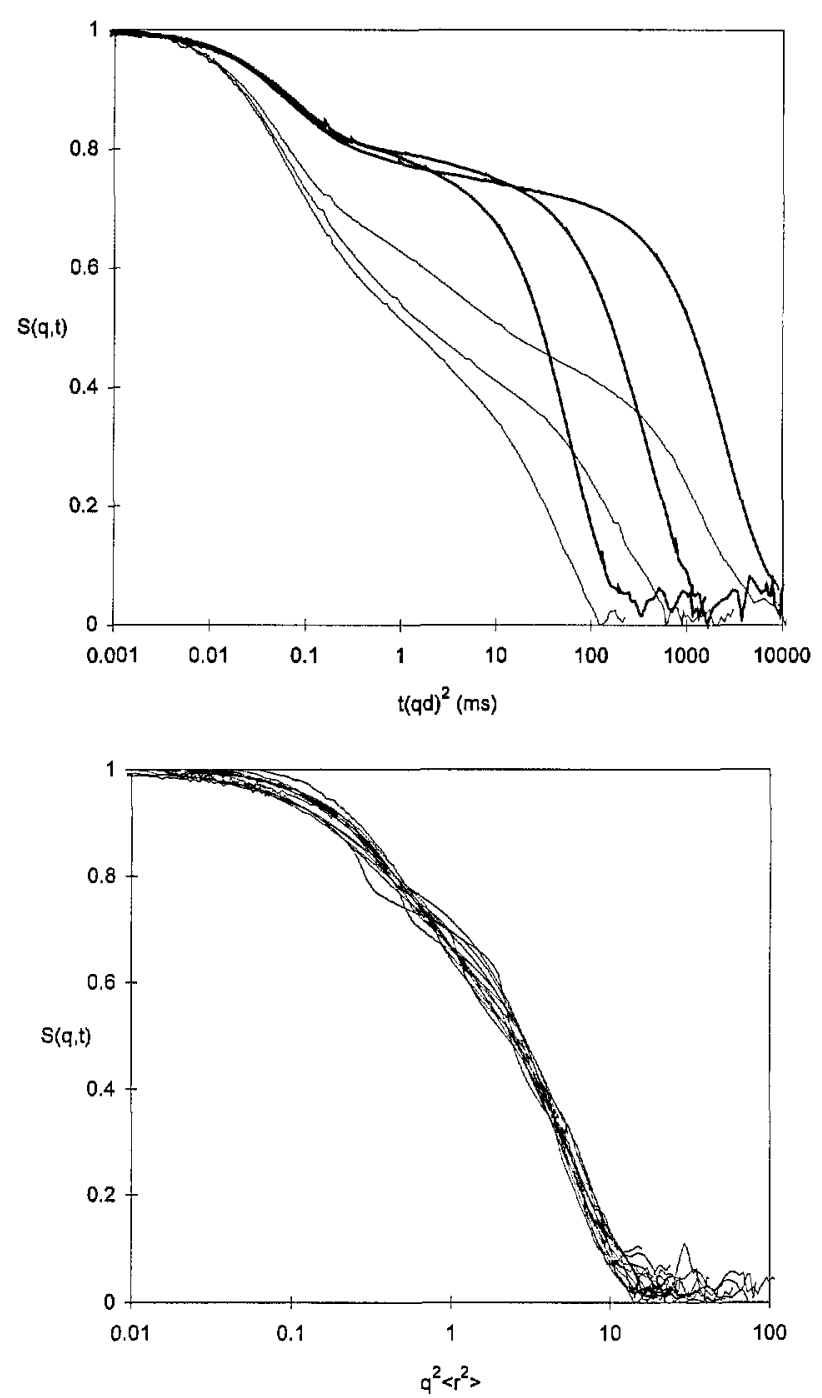

Figure 15. Dynamic structure factor $S(q, t)$ measured at a given concentration $\left(C=0.068 \mathrm{~g} / \mathrm{cm}^{3}\right)$ and three different temperatures $\left(T=30,40\right.$, and $\left.50^{\circ} \mathrm{C}\right):(\mathrm{a}$, top $) S(q, t)$ as a function of $t(q d)^{2}$ for two different transfer vectors $q$ : lines in bold $q / q^{*}=0.16$ and thin lines $q / q^{*}=0.45$. (b, bottom) $S(q, t)$ as a function of $q^{2}\left\langle r(t)^{2}\right\rangle$ for five different transfer vectors $q$ $\left(q / q^{*}=0.16,0.22,0.27,0.32,0.45\right)$, with $\left\langle r(t)^{2}\right\rangle=L^{2}[1$ - exp$\left(-\left(t / t_{1}\right)+\left(t / t_{2}\right)^{\beta}+\left(t / t_{3}\right)\right]$ obtained by optimizing the three independent parameters $t_{1}, t_{2}$, and $\beta$.

reported in section 4: examining the first Bragg peak (Figure 5), it appears that the lower the concentration the softer the structure. More quantitatively, one can see in Figure 10 that the osmotic compressibility varies by a factor 6 for these two concentrations.

Let us come back to the long-time behavior and to relation 13 . The physical meaning of this relation can be clarified as follows. Using the Stokes relation, the long-time diffusion coefficient can be written as $D_{3}=$ $k T /(6 \pi \eta l)$, where $l$ corresponds to the size of the particles performing the collective diffusion process. As long-time scale is concerned, let us assume that the relevant viscosity $\eta$ is the macroscopic viscosity measured by rheology. Thus, eqs 7 and 13 lead to $l \cong d / 10$. This result proceeds from a direct analysis of quasi-elastic light scattering, rheology and small-angle neutron scattering data, without any theoretical model. Thus, it supports $a$ posteriori the initial assumption concerning the elementary events contributing to the collective diffusion of the quasi-elastic model. In brief, quasielastic light scattering experiments amount to the same thing as probing the viscosity with a spherical ball at 


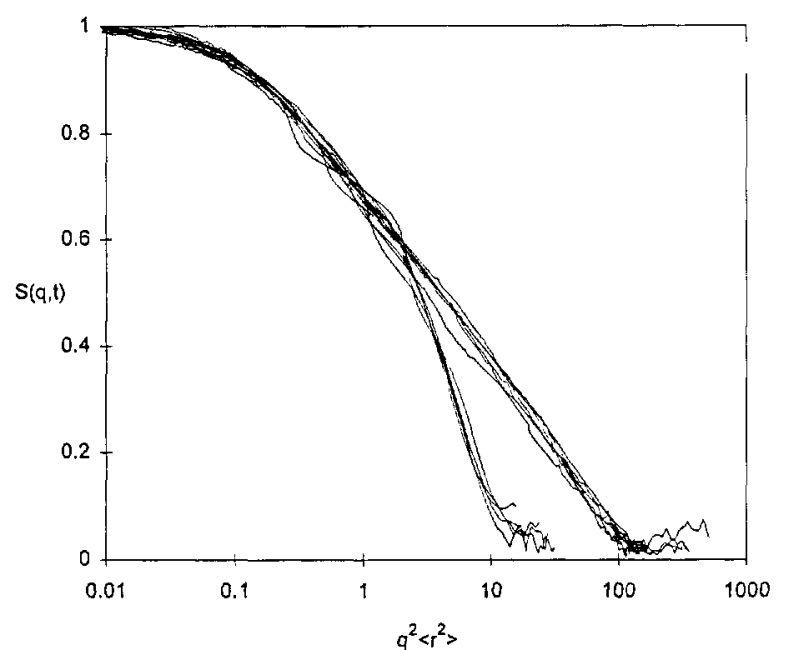

Figure 16. Comparison of the dynamic structure factor master curves $S(q, t)$ vs $q^{2}\left\langle r(t)^{2}\right\rangle$ obtained for two different concentrations: upper master curve $C_{1}=0.034 \mathrm{~g} / \mathrm{cm}^{3}, T=30$ ${ }^{\circ} \mathrm{C}$ and $q / q^{*}=0.20,0.28,0.34,0.40$, and 0.57 ; lower master curve $C_{2}=0.068 \mathrm{~g} / \mathrm{cm}^{3}, T=30^{\circ} \mathrm{C}$ and $q / q^{*}=0.16,0.22,0.27$, 0.32 , and 0.45 .

different time scale, ${ }^{28,29}$ from the solvent viscosity $\left(D_{1}\right.$, $\left.t_{1}\right)$ to the macroscopic viscosity $\left(D_{3}, t_{3}\right)$.

\section{Conclusion and Prospects}

The experimental results reported in this paper emphasize the particular behavior of this copolymersolvent system. The global understanding of its complex behavior passes through the use of several complementary experimental techniques. From the rheological point of view, these semidilute solutions behave like a viscoelastic liquid having a zero shear viscosity and a longest relaxation time that increases with the temperature following a Vogel-Fulcher. law. The plateau modulus corresponds to the one of a network made of end-linked copolymers: $G=k T C / M$. Both the viscous and the elastic behaviors can only be explained by a copolymer transient network made of triblocks linked together by their polystyrene terminal blocks. Smallangle neutron scattering experiments have revealed that this network is well ordered and has a cubic structure, because up to five Bragg peaks have been observed. The relaxation function of the concentration fluctuation measured by quasi-elastic light scattering is somewhat reminiscent of supercooled liquid and colloids near the glass transition. It is interpreted in terms of polystyrene node cooperative diffusion that becomes anomalous at long times (low $q$ ). The confrontation of the quasi-elastic light scattering data with rheological and neutron scattering measurements has pointed out that the long-time cutoff of the dynamical structure factor depends simply on the concentration and the temperature through the ratio of the square lattice unit cell dimension $d^{2}$ to the longest rheological time $\tau_{R}$.

As for the dilute regime, a comparison between the diblock and triblock copolymer behaviors would be fruitful. Preliminary experiments show that diblock copolymers also form structure ir: semidilute solutions. However, in the case of diblock copolymers, the polystyrene cores of the micelles being not connected, the rheological properties behave quite differently. In this context, the study of the dynamius of these systems by quasi-elastic light scattering would be interesting.

Acknowledgment. We thanz J.-P. Bouchaud and C. Safinya, who helped us sort out the quasi-elastic light scattering and neutron scattering results. We are especially grateful to J.-P. Cottcn, who introduced us to the time-of-flight technique.

\section{References and Notes}

(1) Leibler, L. Macromolecules 1980, 13, 1602.

(2) Tuzar, Z.; Kratochvil, P. Surf. Colloid Sci. 1992, 15, 1.

(3) Halperin, A.; Tirrell, M.; Lodge, T. P. Adv. Polym. Sci. 1992, $100,31$.

(4) Raspaud, E.; Lairez, D.; Adam, M.; Carton, J.-P. Macromolecules 1994, 27, 2956.

(5) Adam, M.; Delsanti, M.; Pieransky, P.; Meyer, R. Rev. Phys. Appl. 1984, 19, 253.

(6) Raspaud, E.; Lairez, D.; Adam, M ; Carton, J.-P., in preparation.

(7) Clark, A. H.; Ross Murphy, S. B. Adv. Polym. Sci. 1987, 83, 57.

(8) Adam, M.; Lairez, D. Sol-gel transition. In Physical properties of polymeric gels; Cohen Added, J. P., Ed.; John Wiley \& Sons: Chichester, U.K., in press.

(9) Ferry, J. D. Viscoelastic properties of polymers, 4rd ed.; J. Wiley \& Sons Inc.: New York, 1980.

(10) Adam, M.; Lairez, D.; Raspaud, E. J. Phys. 2 Fr. 1992, 2, 2067.

(11) Raspaud, E.; Lairez, D.; Adam, M. Macromolecules 1995, 28, 927.

(12) Semenov, A. N.; Joanny, J.-F.; Khokhlov, A. R. Macromolecules 1995, 28, 1066.

(13) Cotton, J. P.; Teixeira, J. Physica 1986, 13B, 103.

(14) Nallet, F.; Laversanne, R.; Roux, D. J. Phys. 2 Fr. 1993, 3, 487.

(15) Skouri, M.; Marignan, J.; Appell, J.; Porte, G. J. Phys. 2 Fr. $1991,1,1101$.

(16) ten Brinke, G.; Hadziioannou, G. Macromolecules 1987, 20, 486.

(17) Wang, Y.; Mattice, W. L.; Napprer, D. H. Macromolecules 1992, 25, 4073 .

(18) Adam, M.; Fetters, L. J.; Graes sley, W. W.; Witten, T. A. Macromolecules 1991, 24, 2434.

(19) Wang, C. H.; Fischer, E. W. J. Chem. Phys. 1985, 82, 632.

(20) Meier, G.; Hagenah, J.-U.; Wanc; C. H.; Fytas, G.; Fischer, E. W. Polymer 1987, 28, 1640 .

(21) van Megen, W.; Pusey, P. N. Phys. Rev. A 1991, 43, 5429.

(22) van Megen, W.; Underwood, S. N.. Phys. Rev. Lett. 1993, 70, 2766.

(23) Kob, W.; Andersen, H. C. Phys. Rev. Lett. 1994, 73, 1376.

(24) Gotze, W. In Liquids, freezing and glass transition; Hansen, J. P., Levesque, D., Zinn-Justin, J., Eds.; North Holland: New York, 1989.

(25) Odagaki, T.; Matsui, J.; Hiwata:i, Y. Physica A 1994, 204, 464.

(26) Bouchaud, J. P. J. Phys. 1 Fr. 1992, 2, 1705.

(27) Bouchaud, J. P.; Dean, D. S. Asing on Parisi's tree; SPEC Saclay preprint, 1994.

(28) de Gennes, P. G. J. Phys. Lett. 1979, 40, 197.

(29) Langevin, D.; Rondelez, F. Polyrner 1978, 19, 875

(30) Pedersen, J. S.; Posselt, D.; Mortensen, K. J. Appl. Crystal$\log r . \mathbf{1 9 9 0}, 23,321$.

MA951172X 